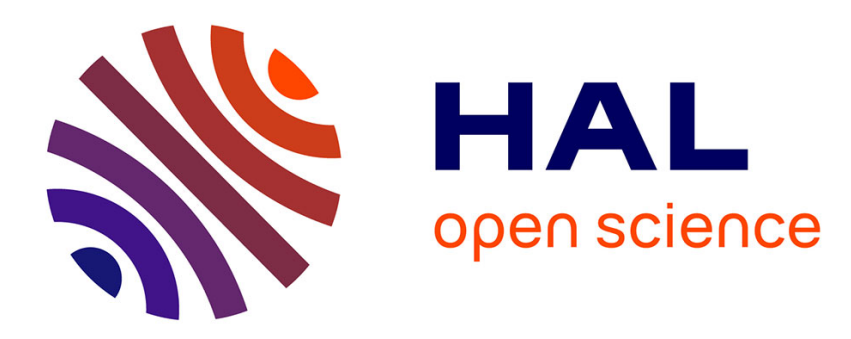

\title{
Clôture intégrale d'idéaux et la propriété $\left(Z_{-} k\right)$. Charef Beddani
}

\section{To cite this version:}

| Charef Beddani. Clôture intégrale d'idéaux et la propriété $\left(Z \_k\right) . .2007$. hal-00165882v2

\section{HAL Id: hal-00165882 \\ https://hal.science/hal-00165882v2}

Preprint submitted on 13 Aug 2007

HAL is a multi-disciplinary open access archive for the deposit and dissemination of scientific research documents, whether they are published or not. The documents may come from teaching and research institutions in France or abroad, or from public or private research centers.
L'archive ouverte pluridisciplinaire HAL, est destinée au dépôt et à la diffusion de documents scientifiques de niveau recherche, publiés ou non, émanant des établissements d'enseignement et de recherche français ou étrangers, des laboratoires publics ou privés. 


\title{
CLÔTURE INTÉGRALE D’IDÉAUX ET LA PROPRIÉTÉ $\left(Z_{k}\right)$
}

\author{
C. BEDDANI
}

\begin{abstract}
Résumé. Dans cet article nous donnons quelques applications du théorème de valuations de Rees à l'étude de la clôture intégrale des idéaux. En particulier, nous étudions la question de Hübl et Swanson (Cf. [2], Question 2.9). Ensuite, nous introduisons une nouvelle propriété des idéaux, que nous l'appelons $\left(Z_{k}\right)$. Cette propriété nous permette de majorer le nombre de valuations de Rees associées à un idéal $I$.
\end{abstract}

\section{INTRODUCTION.}

En appliquant le théorème de valuations de Rees (Cf. Théorème 1.1) à l'étude de la clôture intégrale des idéaux, nous allons montrer le critère suivant :

Critère 1. Soit $I$ un idéal d'un anneau nœthérien $R$, tel que pour tout entier naturel $n \geq 0$, et pour tout $x \in R$, on a : $x^{2} \in I^{2 n+1} \Rightarrow x \in I^{n+1}$. Alors $I$ est normal.

Et nous montrons que la réciproque est vraie (Cf. Proposition 2.4) dans le cas où pour toute valuation de Rees $v$ associée à $I$, on a : $v(I)=1$. De manière semblable et toujours dans le cas où pour toute valuation de Rees $v$ associée à $I$, on a : $v(I)=1$, nous allons montrer un autre critère pour savoir si l'idéal I est intégralement clos ou non. Ce critère s'annonce comme suit :

Critère 2. Soit $I$ un idéal d'un anneau nœthérien $R$, tel que pour toute valuation de Rees $v$ associée à $I$, on a $: v(I)=1$. Alors $I$ est intégralement clos si, et seulement si, pour tout $x \in R$, on a : $x^{2} \in I \Longrightarrow x \in I$.

Par ailleurs, nous allons présenter des diverses résultats concernant la question de Hübl et Swanson (Cf. [2], Question 2.9). Autrement dit, nous allons étudier la question suivante : 
Question 1. Soient $R$ un anneau nœthérien analytiquement irréductible (Cf. Définition 1.3) et $I$ un idéal m-primaire de $R$. Supposons que pour tout entier naturel $n \geq 0$ et pour tous $x$ et $y$ de $R$, on a : $x y \in I^{2 n} \Longrightarrow x \in I^{n}$ ou $y \in I^{n}$ (en particulier, pour tout $x$ de $R$, on a : $x^{2} \in I^{2 n} \Longrightarrow x \in I^{n}$ ). L'idéal $I$ est-il normal?

Ensuite, nous allons donner un cas particulier de certains idéaux dans lesquels cette question a une réponse affirmative. Plus précisément, nous allons montrer le résultat suivant :

Proposition 1. Soient $I$ un idéal de $R$ tel que pour toute valuation de Rees $v_{i}$ associée à $I$, on a : $v_{i}(I)=1$. Alors $I$ est normal si, et seulement si, pour tout $x \in R$, et pour tout entier naturel $n \geq 1$, on a :

(a) $v_{I}(x) \in \mathbb{Z} \Longrightarrow v_{I}\left(x^{2}\right) \in 2 \mathbb{Z}$.

(b) $x^{2} \in I^{2 n} \Longrightarrow x \in I^{n}$.

Enfin, nons allons démontrer le résultat principal de cet article (Cf. Théorème 4.7) qui sert majorer le nombre de valuations de Rees associées à un idéal, qui possède la propriété $\left(Z_{k}\right)$ (Cf. Définition 1$)$. Nous définissions la propriété $\left(Z_{k}\right)$ comme suit :

Définition 1. Soient $I$ un idéal d'un anneau nœthérien $R$ et $k$ un entier naturel supérieur ou égal à 2 . On dit que $I$ possède la propriété $\left(Z_{k}\right)$, s'il existe un entier naturel $b \geq 0$, tel que pour tous $x_{1}, x_{2}, \ldots, x_{k}$ de $R$, et pour tout entier naturel $n \geq 1$, les deux conditions suivante sont vérifiées :

$$
\prod_{i=1}^{k} x_{i} \in I^{k n+b} \Longrightarrow \exists i \in\{1, \ldots, k\}: x_{i} \in I^{n} .
$$

Le résultat principal s'annonce comme suit :

Théorème 1. Soient $(R, \mathfrak{m})$ un anneau nœthérien, $k$ un entier naturel supérieur ou égal à 2 , et $I$ un idéal de $R$ qui possède la propriété $\left(Z_{k}\right)$. Alors $I$ a au plus $(k-1)$ valuations de Rees associées.

A l'aide de ce théorème, nous déduisons une généralisation du critère "One-fiberedness" (Cf. [2], Propositions 2.8). Plus précisément, nous allons montrer le critère suivant :

Critère 3. Soient $R$ un anneau local analytiquement non-ramifié et $I$ un idéal de $R$. Alors $I$ possède la propriété $\left(Z_{2}\right)$ si, et seulement si, $I$ a exactement une seule valuation de Rees associée. 
Les résultats de cet article font partie d'un chapitre de ma thèse, au sein Laboratoire Eimile Picard, sous la direction de M. SPIVAKOVSKY.

Remerciement : je remercie M. SPIVAKOVSKY, pour les remarques et les conseils qui m'ont permis d'apporter certaines précisions et de rendre plus claire plusieurs parties de cet article.

\section{Préliminaires}

Soient $R$ un anneau nœthérien, $I$ un idéal de $R, x$ un élément non nul dans $R$ et $n$ un entier naturel. Nous rappelons que :

- l'ordre de $x$ dans $I$ est le plus grand entier naturel $r$ qui vérifie $x \in I^{r}$, on le note par $v_{I}(x)$.

- L'ordre réduit de $x$ dans $I$ est le nombre $\bar{v}_{I}(x)$ défini par :

$$
\bar{v}_{I}(x)=\lim _{n \rightarrow+\infty} \frac{v_{I}\left(x^{n}\right)}{n} .
$$

Cette limite existe toujours (Cf. [4], Lemme 1.2).

- Nous notons $\bar{I}$ la clôture intégrale de $I$, c'est-à-dire les éléments $x$ dans $R$ qui vérifient une équation de la forme :

$$
x^{s}=a_{1} x^{s-1}+\ldots+a_{s}
$$

où $a_{k} \in I^{k}$ pour tout $1 \leq k \leq s$.

- Nons notons $\mu_{I}(x)$ le plus grand entier naturel $r$ qui vérifie $x \in \overline{I^{r}}$.

- En fin, nous notons :

$$
\bar{\mu}_{I}(x)=\lim _{n \rightarrow+\infty} \frac{\mu_{I}\left(x^{n}\right)}{n} .
$$

Rappelons ici, le théorème de valuations de Rees :

Théorème 1.1 (Théorème de valuations de Rees, Cf. [5]). Soient $R$ un anneau noethérien et I un idéal de R. Il existe un nombre fini de valuations discrètes $\left\{v_{i}\right\}_{1 \leq i \leq r}$ de $R$ telles que pour tout $x \in R-\{0\}$, on $a$ :

$$
\bar{v}_{I}(x)=\min _{1 \leq i \leq r} \frac{v_{i}(x)}{e_{i}}
$$

où $e_{i}=\min \left\{v_{i}(x)\right.$ te que $\left.x \in I\right\}$.

Remarque 1.2. Les valuations $v_{1}, \ldots, v_{s}$ qui apparaissent dans le théorème précédent sont appelées les valuations de Rees associées à I. Et nous avons les propriétés suivantes (Cf. [4, 5, 7]) :

1) Pour tout entier naturel $n \geq 1$, on $a: I^{n}=\left\{x \in R\right.$ tel que $\left.v_{I}(x) \geq n\right\}$.

2) Pour tout entier naturel $n \geq 1$, on $a: \overline{I^{n}}=\left\{x \in R\right.$ tel que $\left.\bar{v}_{I}(x) \geq n\right\}$. 
3) Pour tout $x \in R$, s'il existe une valuation de Rees $v_{i}$ associée à I tel que $v_{i}(x)$ est fini, alors $\bar{v}_{I}(x)$ est un nombre rationnel.

4) Pour tout $x \in$, on $a: \bar{v}_{I}(x)=\bar{\mu}_{I}(x)$.

5) Pour tout $x \in R$, on a $:\left[\bar{v}_{I}(x)\right]=\mu_{I}(x)$, où $\left[\bar{v}_{I}(x)\right]$ est la partie entière de $\bar{v}_{I}(x)$.

Définition 1.3. Soit $(R, m)$ un anneau local intègre. On dit que $R$ est un anneau analytiquement non-ramifié (resp. analytiquement irréductible) si le complété madique de R est réduit (resp. intègre).

\section{CLôture INTÉgrale des IDÉAuX}

Nous nous intéressons dans cette section à l'étude de la clôture intégrale des idéaux $I$ d'un anneau nœethérien, qui vérifient $v(I)=1$ pour toute valuation de Rees associé à $I$.

Définition 2.1. Un idéal I d'un anneau $R$ est dit normal, si pour tout entier naturel $n \geq 1$, on $a: \overline{I^{n}}=I^{n}$.

Proposition 2.2. Soient $R$ un anneau nœthérien et I un idéal de $R$. Les conditions suivantes sont équivalentes :

1) Pour tous entiers naturels $k \geq 1, n \geq 0$, et pour tout $x \in R$, on $a$ :

$$
x^{k} \in I^{k n+1} \Longrightarrow x \in I^{n+1} \text {. }
$$

2) Il existe un entier naturel $k \geq 1$, tel que pour tout entier naturel $n \geq 0$, et pour tout $x \in R$, on $a$ :

$$
x^{k} \in I^{k n+1} \Longrightarrow x \in I^{n+1} .
$$

3) Pour tout entier naturel $n \geq 0$, et pour tout $x \in R$, on $a$ :

$$
x^{2} \in I^{2 n+1} \Longrightarrow x \in I^{n+1} \text {. }
$$

Démonstration. 1) $\Longrightarrow 2$ ) : est trivial.

$2) \Longrightarrow 3$ ) : soient $x$ un élément de $R$ et $v_{I}(x)=n$, alors $x \in I^{n}-I^{n+1}$. D'après la condition 2), il est clair que $x^{k} \in I^{k n}-I^{k n+1}$ et ceci implique que $v_{I}\left(x^{k}\right)=k n$. Donc pour tout $x \in R$, on a : $v_{I}\left(x^{k}\right)=k v_{I}(x)$. Pour montrer que $\forall x \in R, \forall n \in \mathbb{N}$, on a : $x^{2} \in I^{2 n+1} \Longrightarrow x \in I^{n+1}$, il suffit de montrer que pour tout élément $x \in R$, on a $: v_{I}\left(x^{2}\right)=2 v_{I}(x)$. En utilisant l'égalité $v_{I}\left(x^{k}\right)=k v_{I}(x)$, on peut montrer par récurrence que $v_{I}\left(x^{k^{n}}\right)=k^{n} v_{I}(x)$. Donc

$$
\forall x \in R \quad: \quad \frac{v_{I}\left(x^{k^{n}}\right)}{k^{n}}=v_{I}(x)
$$

En passant à la limite quand $n$ tend vers $+\infty$, on obtient :

$$
\forall x \in R: \bar{v}_{I}(x)=v_{I}(x) .
$$


Donc $v_{I}\left(x^{2}\right)=\bar{v}_{I}\left(x^{2}\right)$, et comme la pseudo-valuation $\bar{v}_{I}$ est homogène, il résulte que $v_{I}\left(x^{2}\right)=2 v_{I}(x)$ pour tout $x \in R$.

$3) \Longrightarrow 1$ ) : supposons que pour tout $x \in R$, on a : $v_{I}\left(x^{2}\right)=2 v_{I}(x)$. Soit $k$ un entier naturel non nul. Prenons $s$ un entier naturel tel que $k<2^{s}$. La condition 3) donne $v\left(x^{2^{s}}\right)=2^{s} v(x)$. D'autre part, on a :

$$
\begin{aligned}
v_{I}\left(x^{2^{s}}\right) & =v_{I}\left(x^{2^{s}-k} x^{k}\right) \\
& \geq v_{I}\left(x^{2^{s}-k}\right)+v_{I}\left(x^{k}\right) \\
& \geq\left(2^{s}-k\right) v_{I}(x)+v_{I}\left(x^{k}\right) .
\end{aligned}
$$

Ce qui donne $v_{I}\left(x^{k}\right) \leq k v_{I}(x)+v_{I}\left(x^{2^{s}}\right)-2^{s} v_{I}(x)=k v_{I}(x)$. Donc pour tout $x \in R$, et pour tout entier naturel $n \geq 1$, on a $: v_{I}\left(x^{k}\right)=k v_{I}(x)$. Or, si $x^{k} \in I^{k n+1}$, alors $k v_{I}(x)=v_{I}\left(x^{k}\right) \geq k n+1$, par suite $v_{I}(x) \geq n+\frac{1}{k}$. Donc $x \in I^{n+1}$.

Proposition 2.3. Si I vérifie l'une des conditions de la proposition précédentes, alors I est normal.

Démonstration. Il est clair que si l'idéal $I$ vérifie l'une des conditions de la proposition précédente, alors pour tout $x \in R$, on a : $\bar{v}_{I}(x)=v_{I}(x)$. Le fait que pour tout entier naturel $n$, on a : $I^{n}=\left\{x \in R\right.$ tel que $\left.v_{I}(x) \geq n\right\}$ et $\overline{I^{n}}=\left\{x \in R\right.$ tel que $\left.\bar{v}_{I}(x) \geq n\right\}$ entraîne immédiatement $\overline{I^{n}}=I^{n}$.

Proposition 2.4. Si pour toute valuation $v_{i}$ de Rees associée à $I$, on $a: v_{i}(I)=1$, alors les conditions suivantes sont équivalentes:

1) I est normal.

2) Pour tous $x \in R$ et $n \in \mathbb{N}$, on $a$ :

$$
x^{2} \in I^{2 n+1} \Longrightarrow x \in I^{n+1} .
$$

Démonstration. 2) $\Longrightarrow 1$ ) : voir la proposition précédente $1) \Longrightarrow 2)$ : supposons que $I$ est normal, et soient $x \in R$ et $n$ un entier naturel.

On a :

$$
\begin{aligned}
x^{2} \in I^{2 n+1} & \Longrightarrow x^{2} \in \overline{I^{2 n+1}} \\
& \Longrightarrow \bar{v}_{I}\left(x^{2}\right) \geq 2 n+1 \\
& \Longrightarrow \min _{1 \leq i \leq s}\left\{v_{i}\left(x^{2}\right)\right\} \geq 2 n+1 \\
& \Longrightarrow \forall i=1, \ldots, s \text { on } \mathrm{a}: v_{i}(x) \geq n+1 \\
& \Longrightarrow \bar{v}_{I}(x) \geq n+1 \\
& \Longrightarrow x \in \overline{I^{n+1}}=I^{n+1} .
\end{aligned}
$$


Proposition 2.5. Si pour toute valuation $v_{i}$ de Rees associée à $I$, on $a: v_{i}(I)=1$, alors les deux conditions suivantes sont équivalentes:

1) Pour tout $x \in R$, on $a: x^{2} \in I \Longrightarrow x \in I$.

2) I est intégralement clos.

Démonstration. 1) $\Longrightarrow 2$ ) : supposons que pour tout $x \in R$, on a : $x^{2} \in I \Longrightarrow$ $x \in I$. Soit $x \notin I$, alors pour tout entier naturel $k \geq 0$, on a : $x^{2^{k}} \notin I$ et ceci donne $v_{I}\left(x^{2^{k}}\right)=0$, par suite $\bar{v}_{I}(x)=0$. Donc $x \notin \bar{I}$.

2) $\Longrightarrow 1$ ) : supposons que $\bar{I}=I$. On a :

$$
\begin{aligned}
x^{2} \in I & \Longrightarrow x^{2} \in \bar{I} \\
& \Longrightarrow \bar{v}_{I}\left(x^{2}\right) \geq 1 \\
& \Longrightarrow \min _{1 \leq i \leq s}\left\{v_{i}\left(x^{2}\right)\right\} \geq 1 \\
& \Longrightarrow \forall i=1, \ldots, s \text { on } \mathrm{a}: v_{i}(x) \geq 1 \\
& \Longrightarrow \bar{v}_{I}(x) \geq 1 \\
& \Longrightarrow x \in \bar{I}=I .
\end{aligned}
$$

\section{La question de Hübl et Swanson}

Soient $R$ un anneau nœthérien analytiquement irréductible et $I$ un idéal m-primaire de $R$. Supposons que pour tout entier naturel $n \geq 0$ et pour tous $x$ et $y$ de $R$, on a : $x y \in I^{2 n} \Longrightarrow x \in I^{n}$ ou $y \in I^{n}$ (en particulier, pour tout $x$ de $R$, on a : $\left.x^{2} \in I^{2 n} \Longrightarrow x \in I^{n}\right)$. L'idéal I est-il normal?

Cette question a été posé par R. Hübl et I. Swanson dans un article intitulé "Discrete valuations centered on local domains" (Cf. [2], Question 2.9). Il est clair que si l'idéal $I$ est normal, alors pour tout entier naturel $n \geq 0$ et pour tout $x$ de $R$, on a : $x^{2} \in I^{2 n} \Longrightarrow x \in I^{n}$, car :

$$
\begin{aligned}
x^{2} \in I^{2 n} & \Longrightarrow x^{2} \in \overline{I^{2 n}} \\
& \Longrightarrow \bar{v}\left(x^{2}\right) \geq 2 n \\
& \Longrightarrow \bar{v}(x) \geq n \\
& \Longrightarrow x \in \overline{I^{n}}=I^{n} .
\end{aligned}
$$

On remarque que si l'idéal $I$ vérifie la propriété : pour tout entier naturel $n \geq 0$, et pour tout $x \in R$, on a : $x^{2} \in I^{2 n} \Longrightarrow x \in I^{n}$, alors pour tout entier naturel $s \geq 1$ l'idéal $I^{s}$ vérifie aussi cette propriété. Donc il suffit de donner une réponse à la question suivante : 
Question 3.1. Soient $R$ un anneau nœethérien analytiquement irréductible et I un idéal $\mathrm{m}$-primaire de $R$. Supposons que pour tout entier naturel $n \geq 1$, et pour tout $x$ de $R$, on $a: x^{2} \in I^{2 n} \Longrightarrow x \in I^{n}$. I est-il intégralement clos ?

Lemme 3.2. Soient $R$ un anneau næthérien et I un idéal de $R$. Si pour tout entier naturel $n \geq 1$, et pour tout $x$ de $R$, on $a: x^{2} \in I^{2 n} \Longrightarrow x \in I^{n}$, alors pour tout $n \geq 0$, on $a: \overline{I^{n+1}} \subset I^{n}$.

Démonstration. Soient $x \in R$ et $v_{I}(x)=s$, alors $x \in I^{s}-I^{s+1}$. Par récurrence on peut montrer que pour tout $k \in \mathbb{N}$ on a $x^{s 2^{k}} \in I^{s 2^{k}}$ et $x^{s 2^{k}} \notin I^{(s+1) 2^{k}}$, ce qui donne $s 2^{k} \leq v_{I}\left(x^{2^{k}}\right) \leq(s+1) 2^{k}-1$. Alors pour tout $k \in \mathbb{N}$, on a : $s \leq v_{I}\left(x^{2^{k}}\right) / 2^{k} \leq(s+1)-\left(1 / 2^{k}\right)$. En passant à la limite quand $k$ tend vers $(+\infty)$, on obtient :

$$
v_{I}(x) \leq \bar{v}_{I}(x) \leq v_{I}(x)+1 .
$$

L'inégalité $\bar{v}_{I} \leq v_{I}+1$ entraîne que pour tout $n \geq 0$, l'inclusion: $\overline{I^{n+1}} \subset I^{n}$.

La proposition suivante donne une réponse affirmative à la question de Hübl et Swanson, dans le cas où, toute valuation de Rees $v_{i}$ associée à $I$, vérifie $v_{i}(I)=1$, et pour tout $x \in R$, on a $: v_{I}(x) \in \mathbb{Z} \Longrightarrow v_{I}\left(x^{2}\right) \in 2 \mathbb{Z}$.

Proposition 3.3. Soient I un idéal d'un anneau nœthérien $R$, tel que pour toute valuation de Rees $v_{i}$ associée à $I$, on $a: v_{i}(I)=1$. Alors I est normal si, et seulement si, pour tout $x \in R$, et pour tout entier naturel $n \geq 0$, les deux conditions suivantes sont vérifiées:

(a) $v_{I}(x) \in \mathbb{Z} \Longrightarrow v_{I}\left(x^{2}\right) \in 2 \mathbb{Z}$.

(b) $x^{2} \in I^{2 n} \Longrightarrow x \in I^{n}$.

Démonstration. Supposons que $I$ est normal. Soit $x$ un élément de $R$. On a déjà vu que : $I$ normal implique la condition $(b)$. Le fait que $I$ est normal et que pour tout $1 \leq i \leq s$, on a : $v_{i}(I)=1$ implique que $v_{I}(x)=\bar{v}_{I}(x)$. Ce qui montre que $v_{I}\left(x^{2}\right)=\bar{v}_{I}\left(x^{2}\right)=2 \bar{v}_{I}(x) \in 2 \mathbb{Z}$. Supposons maintenant que $I$ vérifie les conditions $(a)$ et $(b)$. Soient $x$ un élément de $R$ et $n \geq 1$ un entier naturel. On a :

$$
\begin{aligned}
x \in \overline{I^{n}} & \Longrightarrow \bar{v}_{I}(x) \geq n \\
& \Longrightarrow \bar{v}_{I}\left(x^{2}\right) \geq 2 n
\end{aligned}
$$

Or d'après le lemme 3.2. on sait que $v_{I}(x)+1 \geq \bar{v}_{I}(x)$. Par conséquent $v_{I}\left(x^{2}\right)+1 \geq 2 n$, donc $x^{2} \in I^{2 n}$, car $v_{I}\left(x^{2}\right) \in 2 \mathbb{Z}$. La condition (b) implique que $x \in I^{n}$. Donc $\overline{I^{n}}=I^{n}$.

Définition 3.4. Soient $R$ un anneau nothérien et $k$ un entier naturel. On dit que $R$ possède la propriété $S_{k}$ si pour tout idéal premier $p$ de $R$, on $a$ :

depth $R_{\mathfrak{p}} \geq \inf \{k$, ht $\mathfrak{p}\}$. 
Définition 3.5. Soient $R$ un anneau nothérien et $k$ un entier naturel. On dit que $R$ possède la propriété $R_{k}$, si pour tout idéal premier $\mathfrak{p}$ de $R$ de hauteur inférieure ou égale à $k$, l'anneau $R_{\mathfrak{p}}$ est régulier.

Notation 3.6. Si $A=\bigoplus_{i=0}^{+\infty} A_{i}$ est un anneau gradué et $\mathfrak{p}$ un idéal premier de $A$, on note: $A_{(\mathfrak{p})}=\left\{x \in A_{\mathfrak{p}}\right.$ tel que $\left.\operatorname{deg} x=0\right\}$.

Proposition 3.7. Les conditions suivantes sont équivalentes :

1) L'anneau $\overline{R[I T]} / / \overline{R[I T]}$ possède la propriété $\left(R_{0}\right)$.

2) Pour toute valuation de Rees $v$ associée à $I$ on a $v(I)=1$.

Démonstration. 1) $\Longrightarrow 2$ ) : soit $v$ un valuation de Rees associée a I. Alors il existe un idéal premier $\mathfrak{p} \in$ Spec $\overline{R[I T]}$ de hauteur 1 , minimal sur $I \overline{R[I T]}$ tel que $R_{v}=\overline{R[I T]}_{(\mathfrak{p})}$. Posons $\mathfrak{q}=\mathfrak{p} / I \overline{R[I T]}$, alors ht $\mathfrak{q}=0$. Si l'anneau $\overline{R[I T]} / I \overline{R[I T]}$ possède la propriété $\left(R_{0}\right)$, alors l'anneau

$$
(\overline{R[I T]} / I \overline{R[I T]})_{(\mathrm{q})}=R_{v} / I R_{v}
$$

est intègre, donc $I R_{v} \subset \mathfrak{m}_{v}$ est un idéal premier de $R_{v}$ de hauteur supérieure ou égale à $1, \operatorname{car}(0) \varsubsetneqq I$. Comme la hauteur de $\mathrm{m}$ est 1 , il en résulte $I R_{v}=\mathrm{m}_{v}$. Donc $v(I)=1$.

2) $\Longrightarrow 1)$ : supposons maintenant que pour toute valuation de Rees $v$ associée à $I$, on a $: v(I)=1$. Soit q un idéal premier de $\overline{R[I T]} / I \overline{R[I T]}$ de hauteur zéro. Soit $\mathfrak{p}$ la pré-image de $\mathfrak{q}$ dans $\overline{R[I T]}$. Donc $\mathfrak{p}$ est un idéal premier de $\overline{R[I T]}$ de hauteur 1 tel que $\mathfrak{q}=\mathfrak{p} / I \overline{R[I T]}$. Soit $v$ la valuation de Rees associée $I$ tel que $R_{v}=\overline{R[I T]}_{(\mathfrak{p})}$. Donc $I R_{v}=m_{v}$ car $v(I)=1$. D'après l'égalité (3.1), il est clair que $(\overline{R[I T]} / / \overline{R[I T]})_{(\mathfrak{q})}$ est intègre. Donc $\overline{R[I T]} / / \overline{R[I T]}$ possède la propriété $\left(R_{0}\right)$.

Remarque 3.8. Dire que l'anneau $(\overline{R[I T]} / I \overline{R[I T]})$ possède la propriété $\left(R_{0}\right)$, c'est la même chose de dire qu'il est réduit, car cet anneau possède la propriété $\left(S_{1}\right)$.

\section{LA PROPRiÉté $\left(Z_{k}\right)$}

Nous introduisons dans cette section, une nouvelle propriété des idéaux, qu'on l'appelle $\left(Z_{k}\right)$, et nous présentons des diverses résultats liés à cette propriété.

Définition 4.1. Soient I un idéal d'un anneau nœthérien $R$ et $k$ un entier naturel supérieur ou égal à 2 . On dit que I possède la propriété $\left(Z_{k}\right)$, s'il existe un entier naturel $b \geq 0$, tel que pour tous $x_{1}, x_{2}, \ldots, x_{k}$ de $R$, et pour tout entier naturel $n \geq 1$, on $a$ :

$$
\prod_{i=1}^{k} x_{i} \in I^{k n+b} \Longrightarrow \exists i \in\{1, \ldots, k\}: x_{i} \in I^{n}
$$


Proposition 4.2. Soient I un idéal d'un anneau nothérien $R$ et $k$, $s$ deux entiers naturels supérieurs ou égals à 2. On a :

1) Si I possède les propriétés $\left(Z_{k}\right)$, alors $I^{s}$ possède la propriété $\left(Z_{k}\right)$ pour tout $s \in\{2, \ldots, k\}$.

2) Si I possède les propriétés $\left(Z_{k}\right)$ et $\left(Z_{s}\right)$, alors $I^{s}$ et $I^{k}$ possèdent la propriété $\left(Z_{s k}\right)$.

3) Si I possède la propriété $\left(Z_{k^{2}}\right)$, alors I possède la propriété $\left(Z_{k}\right)$. En générale, s'il existe un entier naturel $r \geq 0$, tel que I possède la propriété $\left(Z_{k^{2}}\right)$, alors I possède la propriété $\left(Z_{k}\right)$.

Démonstration. 1) Supposons que I possède la propriété $\left(Z_{k}\right)$. Alors il existe un entier naturel $b \geq 0$, tel que pour tous $x_{1}, x_{2}, \ldots, x_{k}$ de $R$, et pour tout entier naturel $n \geq 1$, on a :

$$
\prod_{i=1}^{k} x_{i} \in I^{k n+b} \Longrightarrow \exists i \in\{1, \ldots, k\}: x_{i} \in I^{n}
$$

Soit $s \in\{2, \ldots, k\}$, on a :

$$
\begin{aligned}
\prod_{i=1}^{s} x_{i} \in I^{(k n+b)} & \Longrightarrow \prod_{i=1}^{s} x_{i} \in I^{k(s n)+s b} \subseteq I^{k(s n)+b} \\
& \Longrightarrow \exists i \in\{1, \ldots, s\}: x_{i} \in I^{s n},(\text { Cf. } 4.2) .
\end{aligned}
$$

Donc $I^{s}$ possède la propriété $\left(Z_{k}\right)$.

2) Supposons que $I$ possède les propriétés $\left(Z_{k}\right)$ et $\left(Z_{s}\right)$. Alors par définition, il existe deux entiers naturels $b_{1} \geq 0, b_{2} \geq 0$, tels que pour tous $x_{1}, x_{2}, \ldots, x_{k}$, $y_{1}, y_{2}, \ldots, y_{s}$ de $R$, et pour tout entier naturel $n \geq 1$, on a :

$$
\prod_{i=1}^{k} x_{i} \in I^{k n+b_{1}} \Longrightarrow \exists i \in\{1, \ldots, k\}: x_{i} \in I^{n}
$$

et

$$
\prod_{j=1}^{s} y_{i} \in I^{s n+b_{2}} \Longrightarrow \exists j \in\{1, \ldots, k\}: y_{j} \in I^{n}
$$

Pour montrer que $I^{s}$ possède la propriété $\left(Z_{s k}\right)$, il suffit de montrer qu'il existe un entier naturel $c \geq 0$, tel que pour tous $x_{11}, x_{12}, \ldots x_{\text {sk }}$ de $R$, et pour tout entier naturel $n \geq 1$, on a :

$$
\prod_{\substack{1 \leq i \leq s \\ 1 \leq j \leq k}} x_{i j} \in I^{k s^{2} n+c s} \Longrightarrow \exists \alpha \in\{1, \ldots, s\}, \exists \beta \in\{1, \ldots, k\}: x_{\alpha \beta} \in I^{s n} .
$$


Prenons $c$ un entier naturel qui vérifie $c s \geq k b_{2}+b_{1}$. On a :

$$
\begin{aligned}
\prod_{\substack{1 \leq i \leq s \\
1 \leq j \leq k}} x_{i j} \in I^{k s^{2} n+c s} & \Longrightarrow \prod_{j=1}^{k} \prod_{i=1}^{s} x_{i j} \in I^{k\left(s^{2} n+b_{2}\right)+b_{1}} \\
& \Longrightarrow \exists \beta \in\{1, \ldots, k\}: \prod_{i=1}^{s} x_{i \beta} \in I^{\left(s^{2} n+b_{2}\right)}, \text { (Cf. (4.3)) } \\
& \Longrightarrow \exists \alpha \in\{1, \ldots, s\}, \exists \beta \in\{1, \ldots, k\}: x_{\alpha \beta} \in I^{s n} \text {, (Cf. (4.3)). }
\end{aligned}
$$

Donc $I^{s}$ possède la propriété $\left(Z_{s k}\right)$. De manière similaire, nous pouvons montrer que $I^{k}$ possède la propriété $\left(Z_{s k}\right)$.

3) Supposons que $I$ possède la propriété $\left(Z_{k^{2}}\right)$. Alors il existe un entier naturel $b \geq 0$, tel que pour tous $x_{11}, x_{12}, \ldots, x_{k k}$ de $R$, et pour tout entier naturel $n \geq 1$, on a :

$$
\prod_{\substack{1 \leq i \leq k \\ 1 \leq j \leq k}} x_{i j} \in I^{k^{2} n+b} \Longrightarrow \exists i, j \in\{1, \ldots, k\}: x_{i j} \in I^{n} .
$$

Montrons maintenant que $I$ possède la propriété $\left(Z_{k}\right)$. Soient $x_{1}, x_{2}, \ldots, x_{k} \in R$, et $n \geq 1$ un entier naturel, on a :

$$
\begin{aligned}
\prod_{i=1}^{k} x_{i} \in I^{k n+b} & \Longrightarrow \prod_{i=1}^{k} x_{i}^{k} \in I^{k^{2} n+b k} \subseteq I^{k^{2} n+b} \\
& \Longrightarrow \exists i \in\{1, \ldots, k\}: x_{i} \in I^{n} \text {, (Cf. (4.5). }
\end{aligned}
$$

S'il un entier naturel $r \geq 0$ tel que $I$ possède la propriété $\left(Z_{k^{2}}\right)$, alors d'après ce qu'on vient de montrer, nous en déduisons par récurrence que $I$ possède les propriétés $\left(Z_{k^{2-1}}\right),\left(Z_{k^{2-2}}\right), \ldots,\left(Z_{k^{2}}\right),\left(Z_{k}\right)$.

Proposition 4.3. Soit I un idéal d'un anneau næthérien R. Les conditions suivantes équivalentes :

1) I possède la propriété $\left(Z_{2}\right)$.

2) Il existe un entier naturel $s \geq 0$, tel que I possède la propriété $\left(Z_{2^{2}}\right)$.

3) Pour tout entier naturel $s \geq 0$, I possède la propriété $\left(Z_{2^{2^{s}}}\right)$.

Démonstration. 3) $\Longrightarrow 2$ ) : est trivial.

$2) \Longrightarrow 1$ ) : est une conséquence immédiate de la proposition 4.2 . Il suffit de remplacer $k$ par 2 dans 3 ).

1) $\Longrightarrow 3)$ : nous allons utiliser la démonstration par récurrence sur s pour montrer que : I possède la propriété $\left(Z_{2^{2}}\right)$.

$\underline{\text { Si } s=0}$ : il n'y a rien montrer.

La récurrence : supposons que $I$ possède la propriété $\left(Z_{2^{2^{s}}}\right)$. Alors il existe un entier naturel $b_{s} \geq 0$, tel que $x_{1}, \ldots, x_{\left(2^{2^{s}}\right)} \in R$, et pour tout entier naturel 
$n \geq 1$, on a :

$$
\prod_{i=1}^{2^{2^{s}}} x_{i} \in I^{2^{2^{s}} n+b_{s}} \Longrightarrow \exists i \in\left\{1, \ldots, 2^{2^{s}}\right\}: x_{i} \in I^{n} .
$$

Montrons maintenant que $I$ possède la propriété $\left(Z_{2^{2^{s+1}}}\right)$. Posons $b_{s+1}=$ $\left(2^{2^{s}}+1\right) b_{s}$. Soient $n \geq 1$ un entier naturel et $\left(y_{i j}\right)_{\substack{1 \leq i \leq 2^{2 s} \\ 1 \leq j \leq 2^{2^{s}}}} 2^{2^{s+1}}$-éléments de $R$.

On a :

$$
\begin{aligned}
\prod_{\substack{1 \leq i \leq 2^{2^{s}} \\
1 \leq j \leq 2^{2^{s}}}} y_{i j} \in I^{2^{2^{s+1}} n+b_{s+1}} & \Longrightarrow \prod_{1 \leq i \leq 2^{2^{s}}} \prod_{1 \leq j \leq 2^{2^{s}}} y_{i j} \in I^{2^{2^{s}}\left(2^{2^{s}} n+b_{s}\right)+b_{s}} \\
& \left.\Longrightarrow \exists i \in\left\{1, \ldots, 2^{2^{s}}\right\}: \prod_{1 \leq j \leq 2^{2^{s}}} y_{i j} \in I^{2^{2^{s}} n+b_{s}}, \text { (Cf. (4.6) }\right) \\
& \Longrightarrow \exists i, j \in\left\{1, \ldots, 2^{2^{s}}\right\},: y_{i j} \in I^{n} \text {, (Cf. (4.6). }
\end{aligned}
$$

Donc I possède la propriété $\left(Z_{2^{2^{s+1}}}\right)$.

Lemme 4.4. Si I possède la propriété $\left(Z_{k}\right)$, alors il existe un entier naturel $l \geq 1$, tel que pour tout entier naturel $n \geq 0$, on $a: \overline{I^{n+l}} \subseteq I^{n}$.

Démonstration. Soient $x$ un élément non nul de $R$ et $s=v_{I}(x)$. Donc $x \notin$ $I^{s+1}$. Comme I possède la propriété $\left(Z_{k}\right)($ Cf. 4.1$\left.)\right), v_{I}\left(x^{k}\right)<k(s+1)+b$. Par recurrence, on peut montrer que pour tout entier naturel $n \geq 1$, on a :

$$
v_{I}\left(x^{k^{n}}\right)<k^{n}(s+1)+\left(k^{n-1}+\ldots+k+1\right) b=k^{n}(s+1)+\frac{\left(k^{n}-1\right) b}{k-1} .
$$

Donc

$$
\frac{v_{I}\left(x^{k^{n}}\right)}{k^{n}}<(s+1)+\frac{\left(1-1 / k^{n}\right) b}{k-1} .
$$

En passant à la limite quand $n$ tend vers $+\infty$ on obtient :

$$
\bar{v}_{I}(x) \leq v_{I}(x)+\frac{b+k-1}{k-1} .
$$

En prenant $l=\left[\frac{b+k-1}{k-1}\right]+1$, on peut montrer d'après l'équation 4.7 que pour tout entier naturel $n \geq 0$, on a $: \overline{I^{n+l}} \subseteq I^{n}$.

Proposition 4.5. Soient $R$ un anneau næethérien, I un idéal de $R$, et $k$ un entier naturel supérieur où égal à 2. Si I possède la propriété $\left(Z_{k}\right)$, alors $\bar{I}$ possède la propriété $\left(Z_{k}\right)$. En plus, si l'anneau $R$ est analytiquement non-ramifié, alors la réciproque est vraie. 
Démonstration. Supposons que $I$ possède la propriété $\left(Z_{k}\right)$. Alors il existe un entier naturel $b \geq 0$, tel que pour tous $x_{1}, x_{2}, \ldots, x_{k}$ de $R$, et pour tout entier naturel $n \geq 1$, on a :

$$
\prod_{i=1}^{k} x_{i} \in I^{k n+b} \Longrightarrow \exists i \in\{1, \ldots, k\}: x_{i} \in I^{n} .
$$

D'après le lemme précédent, il existe un entier naturel $l \geq 0$ tel que pour tout entier naturel $n \geq 0$, on a :

$$
\overline{I^{n+l}} \subset I^{n}
$$

Posons $d=b+l$. On a :

$$
\begin{aligned}
\prod_{i=1}^{k} x_{i} \in(\bar{I})^{k n+d} & \Longrightarrow \prod_{i=1}^{k} x_{i} \in \overline{I^{k n+d}} \\
& \Longrightarrow \prod_{i=1}^{k} x_{i} \in I^{k n+b},(\text { Cf. }(4.8) \text { ) } \\
& \Longrightarrow \exists i \in\{1, \ldots, k\}: x_{i} \in I^{n} \subseteq(\bar{I})^{n} .
\end{aligned}
$$

Donc $\bar{I}$ possède la propriété $\left(Z_{k}\right)$. Réciproquement, supposons que l'idéal $\bar{I}$ possède la propriété $\left(Z_{k}\right)$. Alors il existe un entier naturel $s \geq 0$, tel que pour tous $x_{1}, x_{2}, \ldots, x_{k}$ de $R$, et pour tout entier naturel $n \geq 1$, on a :

$$
\prod_{i=1}^{k} x_{i} \in(\bar{I})^{k n+s} \Longrightarrow \exists i \in\{1, \ldots, k\}: x_{i} \in(\bar{I})^{n} \text {. }
$$

Si l'anneau $R$ est analytiquement non-ramifié, alors d'après Rees (Cf. [6]), il existe un entier naturel $r \geq 0$ tel que pour tout entier naturel $n \geq 0$, on a :

$$
\overline{I^{n+r}} \subset I^{n} \text {. }
$$

Posons $c=s+k r$. On a :

$$
\begin{aligned}
\prod_{i=1}^{k} x_{i} \in I^{k n+c} & \Longrightarrow \prod_{i=1}^{k} x_{i} \in(\bar{I})^{k(n+r)+s} \\
& \Longrightarrow \exists i \in\{1, \ldots, k\}: x_{i} \in(\bar{I})^{n+r} \subseteq \overline{I^{n+r}} \text {, (Cf. } 44.9 \text { ) } \\
& \Longrightarrow \exists i \in\{1, \ldots, k\}: x_{i} \in I^{n} \text {, (Cf. 4.10). }
\end{aligned}
$$

Donc I possède la propriété $\left(Z_{k}\right)$.

Nous allons montrer le résultat principal de cet article (Cf. Théorème 4.7), qui nous permette de borner le nombre de valuations de Rees associées à un idéal $I$ d'un anneau $R$ nœthérien, qui possède la propriété $\left(Z_{k}\right)$. Pour cela nous aurons besoin du lemme suivant : 
Lemme 4.6. Soient I un idéal d'un anneau næthérien $R$ et $v_{1}, v_{2}, \ldots, v_{s}$ ses valuation de Rees associées. Alors pour tout $i \in\{1,2, \ldots, s\}$, il existe un élément $x_{i} \in R$ tel que pour tout $j \neq i$, on $a$ :

$$
v_{i}\left(x_{i}\right) / e_{i}<v_{j}\left(x_{i}\right) / e_{j},
$$

où $e_{i}=v_{i}(I)$.

Démonstration. Soient pour tout $i \in\{1,2, \ldots, s\}, \mathfrak{p}_{i}$ l'idéal premier homogène de $S=\overline{R[I T, U]} /(U T-1)$ associé à $(U)$ tel que $R_{v_{i}}=S_{\left(p_{i}\right)}$. Notons $\tilde{v}_{i} l^{\prime}$ extension de $v_{i}$ à $S_{\mathfrak{p}_{i}}$. Comme les idéaux $\left(\mathfrak{p}_{i}\right)_{i}$ sont distincts, pour tout $i \in\{1,2, \ldots, s\}$, il existe un élément $h_{i} \notin \mathfrak{p}_{i}$ et $h_{i} \in \bigcap_{j \neq i} \mathfrak{p}_{j}$. Par conséquent $\tilde{v}_{i}\left(h_{i}\right)=0$ et $\tilde{v_{j}}\left(h_{i}\right) \geq 1$ pour tout $j \neq i$. Comme les idéaux $\left(\mathfrak{p}_{i}\right)_{i}$ sont homogènes, on peux considérer que l'élément $h_{i}$ est homogène (ie. $h_{i}=a T^{n}$, avec $a \in \overline{I^{n}}$ ). Nous distinguons trois cas :

$\underline{\text { Si } n=0}$. On peut prendre $x_{i}=a$.

Si $n<0$. On a :

$$
h_{i}=a T^{n}=a T^{n+1} U \in U S \subset \bigcap_{j=1}^{s} \mathfrak{p}_{j},
$$

ceci contredit le fait que $h_{i} \notin \mathfrak{p}_{i}$.

$\underline{\text { Si } n>0}$. On a :

$$
v_{i}(a)=\tilde{v_{i}}(a)=\tilde{v_{i}}\left(a T^{n} U^{n}\right)=\tilde{v_{i}}\left(a T^{n}\right)+n \tilde{v_{i}}(U)=n e_{i} .
$$

D'autre part, pour tout $j \neq i$, on a :

$$
v_{j}(a)=\tilde{v_{j}}(a)=\tilde{v_{j}}\left(a T^{n} U^{n}\right)=\tilde{v_{j}}\left(a T^{n}\right)+n \tilde{v_{j}}(U)>n e_{j} .
$$

Donc, on a bien : $v_{i}(a) / e_{i}<v_{j}(a) / e_{j}$.

Théorème 4.7. Soient $(R, \mathfrak{m})$ un anneau nœthérien, $k$ un entier naturel supérieur ou égal à 2 , et I un idéal de $R$ qui possède la propriété $\left(Z_{k}\right)$. Alors I a au plus $(k-1)$ valuations de Rees associées.

Démonstration. Soient $v_{1}, v_{2}, \ldots, v_{r}$ les valuations de Rees associées à $I$. Supposons que $r \geq k$. Donc d'après le lemme 4.6. pour tout $i \in\{1, \ldots, r\}$, il existe un élément $a_{i} \in R$ tel que pour tout $j \neq i$, on a : $\omega_{i}\left(a_{i}\right)<\omega_{j}\left(a_{i}\right)$, où $\omega_{i}=v_{i} / e_{i}$. On peut choisir les $a_{i}$ dans $R$ tels que $v_{i}\left(a_{i}\right)>0$. Soit

$$
y_{i}=a_{i}^{e_{i} \prod_{j=1, j i i}^{r} e_{j} \omega_{j}\left(a_{j}\right)} .
$$

Donc pour tout $i \in\{1, \ldots, r\}$, et pour tout $j \neq i$, on a : $\omega_{i}\left(y_{i}\right)<\omega_{j}\left(y_{i}\right)$, et

$$
\omega_{1}\left(y_{1}\right)=\cdots=\omega_{r}\left(y_{r}\right)=\prod_{i=1}^{r} e_{i} \omega_{i}\left(a_{i}\right) .
$$


Posons $s=\prod_{i=1}^{r} e_{i} \omega_{i}\left(a_{i}\right)$. Soit $q$ un nombre rationnel tel que pour tout $j \neq i$, on a :

$$
\omega_{i}\left(y_{i}\right)+q \leq \omega_{j}\left(y_{i}\right)
$$

Comme l'idéal I possède la propriété $\left(Z_{k}\right)$, il existe un entier naturel $b$ tel que pour tous $x_{1}, x_{2}, \ldots, x_{k}$ de $R$ et pour tout entier naturel $n \geq 1$, on a :

$$
\prod_{i=1}^{k} x_{i} \in I^{k n+b} \Longrightarrow \exists i \in\{1, \ldots, k\}: x_{i} \in I^{n} .
$$

De plus, d'après le lemme 4.4, il existe un entier naturel $l \geq 0$ tel que pour tout entier naturel $n \geq 0$, on a :

$$
\overline{I^{n+l}} \subset I^{n} .
$$

Prenons $m$ un entier naturel tel que $[m(s(k-1) q)] \geq k+b+l$. On a :

pour tout $i \in\{1, \ldots, k\}$

$$
\begin{aligned}
\omega_{i}\left(y_{1}^{m} y_{2}^{m} \cdots y_{k}^{m}\right) & =m \omega_{i}\left(y_{i}\right)+m \sum_{j=1, j \neq i}^{k} \omega_{i}\left(y_{j}\right) \\
& \geq m s+m \sum_{j=1, j \neq i}^{k}(s+q) \\
& =m s+m(k-1)(s+q) \\
& =k m s+(k-1) m q
\end{aligned}
$$

et pour tout $i \in\{k+1, \ldots, r\}$

$$
\begin{aligned}
\omega_{i}\left(y_{1}^{m} y_{2}^{m} \cdots y_{k}^{m}\right) & =m \sum_{j=1}^{k} \omega_{i}\left(y_{j}\right) \\
& \geq m \sum_{j=1}^{k}(s+q) \\
& =m(k-1)(s+q) \\
& =(k-1) m s+(k-1) m q .
\end{aligned}
$$

Donc pour tout $i \in\{1, \ldots, r\}$, on a :

$$
\begin{aligned}
\omega_{i}\left(y_{1}^{m} y_{2}^{m} \cdots y_{k}^{m}\right) & \geq(k-1) m s+(k-1) m q \\
& \geq k(s m+1)+(m s+(k-1) m q-k) \\
& \geq k(s m+1)+b+l .
\end{aligned}
$$

Ceci montre que $y_{1}^{m} y_{2}^{m} \cdots y_{k}^{m} \in \overline{I^{k(s m+1)+b+l}}$. Donc $y_{1}^{m} y_{2}^{m} \cdots y_{k}^{m} \in I^{k(s m+1)+b}$. Comme I possède la propriété $\left(Z_{k}\right)$, il existe $i \in\{1, \ldots k\}$ tel que $y_{i}^{m} \in I^{(s m+1)}$, ce qui contredit le fait que $\omega_{i}\left(y_{i}^{m}\right)=s m$. 
Corollaire 4.8. Soient $R$ un anneau næthérien analytiquement non-ramifié et I un idéal de R. I possède la propriété $\left(Z_{2}\right)$ si, et seulement si, I a exactement une seule valuation de Rees associée.

Démonstration. D'après le théorème 4.7 , il est clair que si I possède la propriété $\left(Z_{2}\right)$, alors $I$ a une seule valuation de Rees associée. Réciproquement, si $I$ a une seule valuation de Rees associée, alors la fonction $\bar{v}_{I}$ définit une valuation à valeurs dans $\mathbb{Q}$. Posons $b=2 l$ où $l$ est l'entier naturel qui vérifie pour tout $n \geq 0$, l'inclusion $\overline{I^{n+l}} \subset I^{n}$ (Cf. [6]). Soient $x_{1}, x_{2}$ deux éléments de $R$ tels que $x_{1} x_{2} \in I^{2 n+b}$. Alors $\bar{v}_{I}\left(x_{1}\right)+\bar{v}_{I}\left(x_{2}\right)=\bar{v}_{I}\left(x_{1} x_{2}\right) \geq 2 n+b \geq 2(n+l)$, par conséquent $\bar{v}_{I}\left(x_{1}\right) \geq(n+l)$ ou $\bar{v}_{I}\left(x_{2}\right) \geq(n+l)$, par suite $x_{1} \in \overline{I^{n+l}}$ ou $x_{2} \in \overline{I^{n+l}}$. Donc $x_{1} \in I^{n}$ ou $x_{2} \in I^{n}$, car $\overline{I^{n+l}} \subset I^{n}$. Cela montre que $I$ possède la propriété $\left(Z_{2}\right)$.

Remarque 4.9. Ce dernier corollaire est une généralisation du critère "Onefiberedness" introduite dans les travaux de Swanson et Hübl (Cf. [2], Proposition 2.8).

Corollaire 4.10. Soient $(R, \mathfrak{m})$ un anneau noethérien analytiquement irréductible et I un idéal de R. Les conditions suivantes sont équivalentes :

1) I possède la propriété $\left(Z_{2}\right)$.

2) $\widehat{I R}$ possède la propriété $\left(Z_{2}\right)$, où $\widehat{R}$ est le complété m-adique de $R$.

Démonstration. Puisque l'anneau $R$ est analytiquement irréductible, $I$ et $\widehat{I R}$ ont le même nombre de valuations de Rees associées. Donc si $I$ (resp. $\widehat{I R}$ ) possède la propriété $\left(Z_{2}\right)$, alors $I$ (resp. $\left.I \widehat{R}\right)$ a une seule valuation de Rees associée (Cf. Proposition 4.8). Par suit, $I \widehat{R}$ (resp. $I$ ) a aussi une seule valuation de Rees associée. Donc $I R$ (resp. $I$ ) possède la propriété $\left(Z_{2}\right)$ (Cf. Proposition 4.8).

Définition 4.11. Soient I un idéal d'un anneau noethérien, et $v_{1}, v_{2}$ deux valuations de Rees associées à I. On dit que la topologie v $v_{1}$-adique et la topologie $v_{2}$-adique sont linéairement équivalentes (ou $v_{1}$ et $v_{2}$ sont linéairement comparables) si, et seulement si, il existe un entier naturel $r$ tel que pour tout $x \in R$ non nul, on a :

$$
v_{1}(x) \leq r v_{2}(x) \text { et } v_{2}(x) \leq r v_{1}(x) .
$$

Notation 4.12. Soient $v$ une valuation de Rees associée à un idéal I d'un anneau nothérien $R$, et $n$ un entier naturel. On note:

1) $v(I)=\min \{v(x)$ tel que $x \in I\}$.

2) $I_{n}(v)=\{x \in R$ tel que $v(x) \geq n\}$.

Proposition 4.13. Soient $R$ un anneau nœthérien et I un idéal de $R$. Si toutes les valuations de Rees associées à I sont linéairement comparables, alors $\sqrt{I}$ est premier. 
Démonstration. Soient $v_{1}, v_{2}, \ldots, v_{s}$ les valuations de Rees associées à $I$. D'après le théorème de valuations de Rees et la remarque 1.2 , on sait que :

$$
\bar{I}=\bigcap_{i=1}^{s} I_{e_{i}}\left(v_{i}\right), \text { où } e_{i}=v_{i}(I) \text {. }
$$

Donc

$$
\sqrt{\bar{I}}=\bigcap_{i=1}^{s} \sqrt{I_{e_{i}}\left(v_{i}\right)}=\bigcap_{i=1}^{s} I_{1}\left(v_{i}\right) .
$$

Comme toute les valuations de Rees $v_{1}, v_{2}, \ldots, v_{s}$ sont linéairement comparables, on a :

$$
I_{1}\left(v_{1}\right)=I_{1}\left(v_{2}\right)=\cdots=I_{1}\left(v_{s}\right),
$$

donc $\sqrt{\bar{I}}=I_{1}\left(v_{1}\right)$, ce qui montre que $\sqrt{\bar{I}}$ est premier, donc il est de même pour $I, \operatorname{car} \sqrt{I}=\sqrt{I}$.

Corollaire 4.14. Soient $R$ un anneau nœthérien et I un ideal de R. Si $\sqrt{I} n^{\prime}$ est pas premier, alors I a au moins deux valuations de Rees non-linéairement comparables.

Proposition 4.15. Soient $R$ un anneau nœthérien analytiquement non-ramifié et I un idéal de $R$ tel que son radical n'est pas premier. Si I possède la propriété $\left(Z_{3}\right)$, alors I a exactement deux valuations de Rees associées.

Démonstration. Cette proposition est une conséquence immediate du théorème 4.7 et du corollaire 4.14.

\section{RÉFÉRENCES}

[1] D. Delfino and I. Swanson. Integral closure of ideals in excellent local rings. J. Algebra, 274(1) :422-428, 2004.

[2] R. Hübl and I. Swanson. Discrete valuations centered on local domains. J. Pure Appl. Algebra, 161(1-2) :145-166, 2001.

[3] M. Nagata. Local rings. Interscience Publishers. Interscience Tracts in Pure and Applied Mathematics, 1962.

[4] D. Rees. Valuations associated with a local ring (I). J. London Math. Soc., 5 :108-128, 1955.

[5] D. Rees. Valuations associated with ideals. II. J. London Math. Soc., 31 :221-228, 1956.

[6] D. Rees. A note on analytically unramified local rings. J. London Math. Soc., 36 :24-28, 1961.

[7] D. Rees. Izumi's theorem. In Commutative algebra, volume 15 of Math. Sci. Res. Inst. Publ., pages 407-416. Springer, New York, 1989. 
Charef BEDDANI

Département de Mathématiques

Laboratoire Emile Picard

Université Paul Sabatier

31068 Toulouse - FRANCE

beddani@picard.ups-tlse.fr 